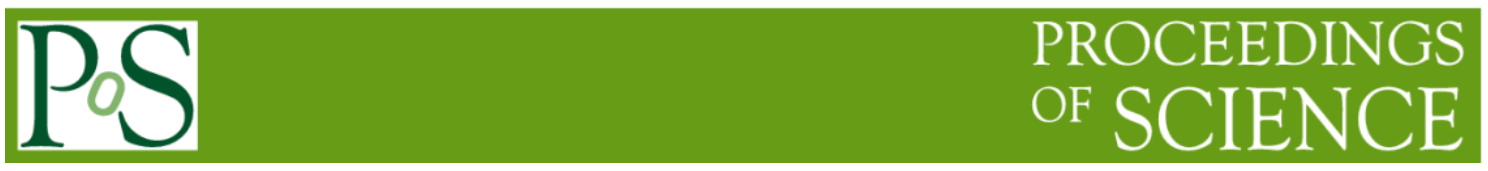

\title{
Opening talk
}

\section{Zdeněk Doležal*}

Charles University, Faculty of Mathematics and Physics

V Holešovičkách 2, Prague, The Czech Republic

E-mail: doleza@ipnp.mff.cuni.cz

This is the opening talk of the conference given by the International Advisory Committee chair.

40th International Conference on High Energy physics - ICHEP2020

July 28 - August 6, 2020

Prague, Czech Republic (virtual meeting)

"Speaker

(C) Copyright owned by the author(s) under the terms of the Creative Commons 
Ladies and gentlemen, dear colleagues, dear guests,

It is my great privilege to welcome you at the 40th International Conference on High Energy Physics

This conference, dubbed also the big Rochester, has become throughout its 70-year history undoubtedly the leading conference in our field. Its beginning was quite modest, though. The first Rochester, organised by Robert Marshak at the University of Rochester, USA in 1950, was a one-day meeting hosting 25 participants. It took years till this conference became larger and international. Even if the number of participants grew only slowly, the meetings contributed hugely to the exchange of information in the pre-electronic era. Just one example: it was here where the regular summaries of particle masses, lifetimes, and other properties became collected and gave birth to the famous PDG booklets.

Recent decades saw ICHEP growing in all parameters. Introducing parallel sessions opened up the chance to attend to many younger researchers who could enjoy the presence of famous colleagues and listen to their rapporteur talks and summaries. The geographical pattern from the sixties 'USA - Western Europe - Soviet Union' was extended to cover all regions, and the organisation of the conference was overseen by the IUPAP C11 Commission on Particles and Fields.

It was 4 years ago when the 2020 edition of ICHEP was assigned to Prague. The typical attendance now has increased to above 1000 and the duration to over a week, so this meeting can no longer be organised by a single person. Instead, the whole particle physics community in the Czech Republic, coming from the 5 universities and 2 research institutes, joined forces and started the preparations. Let me list then here: Charles University, Czech Technical University in Prague, Institute of Physics of the Czech Academy of Sciences, Nuclear Physics Institute of the Czech Academy of Sciences, Palacky University, Olomouc, Technical University Liberexc and University of Western Bohemia Pilsen. We have worked on the scientific programme, social events, and outreach, as well as the diversity and inclusivity aspects, looking forward to have you here.

However in March the circumstances changed dramatically and after many discussions ICHEP2020 moved to this online format. Now it has become obvious that it was the only alternative to a full cancellation. Yet we are aware that this format can never satisfy all the expectations and serve all the roles of the in-person conference. It is a huge experiment - let's view it like that. And as in any experiment it is the joint effort of all of the participants that is the key to success. The number of registrants reached 3000 - quite a familiar number to an LHC physicist. Seventeen parallel sessions that ran last week, and 4 days of Plenaries and discussion panels this week pose many challenges but I believe they will show strength of our community.

Here I have to thank the C11 commission: we have been given a second chance: ICHEP2024 has been assigned to Prague and let's hope it will be a standard one. 
My last comment is on the scientific program. Let me quote here the words of John Polkinghorne, theorist, historian of physics, and author of a great book, "Rochester Roundabout" [1], from 1989:

'Many say, there is a desert region between electroweak unification and grand unification in which nothing really interesting happens... A sad thought, you might think. Take courage. The lessons of history are against such a view. It would be astonishing indeed if the future did not have some surprises in store.' I personally hope this store opens from time to time and nature keeps bringing some surprises, at least until 2024. Let me thank you very much for attending in an unprecedented number, and wish you an enjoyable and fruitful conference!

\section{References}

[1] J. C. Polkinghorne, Rochester Roundabout: The Story of High Energy Physics, W H Freeman \& Co, 1989 